\title{
Cadena productiva del café en el departamento de Nariño
}

Análisis de un caso exitoso

\author{
Silvio A. Delgado U. ${ }^{1}$
}

Alicia Cristina Silva $C^{2}$.

Oscar Eduardo Sanclemente R. ${ }^{3}$

\section{Resumen}

Sobre el origen del cultivo del café en Colombia existen controversias, sin embargo, la versión más aproximada es aquella que señala que fue un grupo de monjes quienes introdujeron el arbusto al Departamento del Cauca en donde no tuvo mucha acogida, situación contraria sucedió con los colonos del eje cafetero quienes encuentran mayores atractivos y ventajas para cultivarlo y comercializarlo.

Lo cierto es que en Colombia después del petróleo el café es el segundo producto con grandes cantidades de exportación, situación que implica cambios frecuentes en el mundo global que obliga a productores y comercializadores a cultivar y transformar un café de calidad con características diferenciadoras que lo hagan atractivo para los consumidores.

Sin embargo, para la Federación Nacional de Cafeteros el café no solamente es un producto agrícola, sino que tiene mucho que ver con el tejido social, cultural y político que inclusive ha servido para la estabilidad democrática y la integridad nacional.

\footnotetext{
1 Administrador público, especialista en Educción cultura y política, Magíster en Administración de organizaciones. Docente Universidad Nacional Abierta y a Distancia - UNAD. silvio.delgado@unad.edu.co

${ }^{2}$ Administradora de empresas, especialista en Pedagogía para el Desarrollo del Aprendizaje Autónomo. Magíster en Sistemas de Calidad y Productividad.Estudiante de Doctorado de Administración de Negocios, mención Gerencia. Docente Universidad Nacional Abierta y Distancia - UNAD. alicia.silva@unad.edu.co.

${ }^{3}$ Ingeniero Ambiental, Magister en Ciencias Agrarias, Doctor en Agroecología, Docente Universidad Nacional Abierta y a Distancia - UNAD. oscar.sanclemente@unad.edu.co
} 
Por su parte, en Nariño treinta y cinco de los municipios de los sesenta y dos que tiene el departamento se dedican al cultivo del café, cultivos al cuidado de las familias que le imprimen toda su atención y esmero en pro de ofrecer cafés de alta calidad dignos de reconocimiento en el mundo como lo demuestran una variedad de premios obtenidos registrados por la Cooperativa de Caficultores del Occidente de Nariño Ltda.

Nariño es un gran productor de cafés especiales un $98 \%$ de los cafés producidos en Nariño, son considerados especiales; situación que se deriva de la gran labor de asesoría que cumple la Cooperativa de Caficultores del Occidente del Departamento de la mano con la Federación Nacional de cafeteros de Colombia y que hace de la cadena productiva del café un caso exitoso digno de analizar.

Palabras claves: Café, Cooperativa, calidad, Caficultor, Nariño

\section{Introducción}

El departamento de Nariño situado al sur de Colombia tiene 62 municipios de los cuales 35 de ellos se dedican al cultivo del café gracias a que el territorio posee todos los pisos térmicos. Los caficultores del departamento han centrado sus esfuerzos en producir cafés de calidad que les permita alcanzar niveles competitivos altos y que se evidencia en el logro de varios premios nacionales e internacionales obtenidos.

El siguiente documento analiza un caso exitoso de la cadena productiva del café en Nariño desde la parte histórica, la importancia del cultivo para los nariñenses y la experiencia exitosa por parte de la Cooperativa de Caficultores del Occidente de Nariño Ltda.

\section{La historia}


Hablar de la cadena productiva del café, es remitirse a la historia misma del producto. Un vegetal que según los entendidos proviene de la antigua Arabia hoy República de Yemen y en donde aún se lo puede encontrar en estado natural y silvestre. Sobre las rutas de propagación se originan muchas leyendas; es así que gracias a la magia de las nuevas tecnologías de la información y la comunicación se puede tener acceso a ese mundo fascinante de la historia de este apreciado y aromático arbusto. Al respecto, en el sitio de Café Kina.com, (sf) señala que:

Durante el siglo XVII, los colonizadores europeos comenzaron a llevar y cultivar el cafeto en sus colonias [...] Los holandeses llevaron sus primeros pies de cafeto a Surinam (Guyana Holandesa) en 1718, y dieron vía para que solo unos años después se creara el primer cafetal brasileño [...] Los ingleses llevaron en 1730 el café a la isla de Jamaica y comenzaron el cultivo del famoso Blue Mountain Coffee. 20 años después se empezó a cultivar café en Guatemala, luego en Cuba y Costa Rica, hasta legar en 1790 a México, en 1825 a Hawái, en 1887 a Vietnam para finalmente llegar en 1896 a Australia, completando así, la vuelta al mundo, el apetecido y fabuloso cafetal.

Con relación a Colombia, que entre otras ostenta el café más suave del mundo, para la Federación de cafeteros (2010) su llegada es también aún controvertida, sin embargo la mayor aproximación que existe al respecto, es que fue introducido por una comunidad de monjes al Departamento del Cauca en donde no tuvo mucho auge hasta que se concentró en Antioquia y el eje cafetero y fueron los colonos quienes encuentran en el producto maneras acertadas de cultivarlo y comercializarlo;de allí en adelante, el café pertenece a la tradición y a la cultura de los colombianos; tanto así, que del deleite 
de una taza de café surgen los amigos, los cuentos fantásticos de los abuelos, nacen las notas inspiradoras de los músicos, las románticas estrofas de los versos que enamoran; por que el café es inspirador de arte en todo el sentido de la palabra.

\section{Importancia del café}

Visto así, El café más que un producto agrícola es ante todo un tejido social, cultural, institucional y político que ha servido de base para la estabilidad democrática y la integridad nacional. Esta actividad representa el corazón de la sociedad rural colombiana ofreciendo una oportunidad de trabajo, de ingreso y de subsistencia". Sin duda, estas palabras extractadas del Informe Final de la Comisión de Ajuste de la Institucional Cafetera (2002) son las que mejor resumen la importancia del café para la economía del país.

\section{El café en Nariño}

Dejando a un lado la historia y todas aquellas connotaciones llenas de virtudes cafeteras, es preciso adentrarnos concretamente en la importancia del grano para el Departamento de Nariño. Al respecto, un documento emanado de la gobernación del Departamento (2002) señala:

La integración gremial, la conformación de una empresa de economía mixta y la certificación del café de Nariño son algunas de las metas de la Secretaría de Agricultura y la Gerencia de la Subregión Norte, constituida en el actual Gobierno Departamental. Con la estrategia se pretende cumplir con el objetivo de generar mayores ingresos para los nariñenses.

Por su parte, el Plan de desarrollo del Departamento en los aspectos generales del sector agrícola en su dimensión económica, contempla Impulsar a las cadenas productivas detectadas (comercio, ecoturismo, artesanías, palma, cacao, forestales, pesqueros, café, caña panelera, lácteos y papa). Concretamente con la 
cadena productiva del café señala la importancia de impulsar el estudio de mercadeo para la construcción de una empresa fabricadora de café tostado y molido.

\section{Una experiencia exitosa}

Así las cosas, sin duda alguna, unos de los casos exitosos de la cadena productiva del café en el Departamento, lo protagoniza la Cooperativa de Caficultores del Occidente de Nariño Ltda. y a la cual pertenece el Ingeniero Agro Industrial Carlos Oliver Oliva Ortega quien se desempeña como Fiel de Compras Sede Pasto, analista de Calidad, y catador de café certificado como Juez de Catación por la Asociación de Cafés Especiales de América (SCAA) en Long Beach California, en el año 2004.

Como gran conocedor de los temas relacionados a la cadena productiva del café en Nariño, el Ingeniero Oliva al preguntarle: por qué considera a la Cooperativa de Caficultores del Occidente de Nariño Ltda. un caso exitoso, responde:

"La Cooperativa es considerada un éxito dentro de la cadena productiva del café, por que ha cumplido y a alcanzado sus objetivos y sus metas; a lo largo de sus treinta y tres años, ha ido creciendo paso a paso desde su fundación el primero de marzo de 1977 con aproximadamente cincuenta asociados distribuidos en Sandoná y Pasto. Inicialmente, la Cooperativa funcionaba en el municipio de Sandoná y posteriormente se fue extendiendo dadas las inquietudes de otros municipios del occidente por formar parte de la Cooperativa hasta centralizar definitivamente su accionar en la capital del Departamento".

La Cooperativa a través de la Federación Nacional de Cafeteros ha comercializado cafés especiales por los cuales ha recibido una bonificación adicional que ha sido distribuida a los caficultores que le venden su producción a la Cooperativa 
El programa de compra y comercialización de cafés especiales en la Cooperativa de Caficultores del Occidente de Nariño inicia con los siguientes tipos de cafés:

Tabla 1. Cafés especiales en Nariño

\begin{tabular}{|c|c|c|c|c|}
\hline Año & Tipo de café & Municipios & \multicolumn{2}{|c|}{ Destino } \\
\hline \multirow[t]{3}{*}{2.001} & "Esperanzas de Nariño" & Sandoná & & \\
\hline & & Samaniego & EEUU, & JAPÓN, \\
\hline & & La Florida & \multicolumn{2}{|c|}{ ALEMANIA } \\
\hline \multirow[t]{6}{*}{2.002} & Esperanzas de Nariño & & & \\
\hline & Illycaffe & Municipios & EEUU, & JAPÓN, \\
\hline & & Occidente de Nariño & \multicolumn{2}{|c|}{ ALEMANIA } \\
\hline & & & \multicolumn{2}{|c|}{ ITALIA } \\
\hline & Plante Nariño & Andes- & & \\
\hline & & Samaniego & & \\
\hline \multirow[t]{5}{*}{2.003} & Regional Nariño & Municipios & & \\
\hline & Illycaffe & Occidente de Nariño & EEUU, & JAPÓN, \\
\hline & & Andes-Samaniego & \multicolumn{2}{|c|}{ ALEMANIA } \\
\hline & Plante Nariño & Consacá & \multicolumn{2}{|c|}{ ITALIA } \\
\hline & ACDI/VOCA & & & \\
\hline
\end{tabular}

Fuente: Cooperativa de Caficultores de Occidente de Nariño Ltda.

Por otra parte, En el año 2.002, la firma italiana Illycaffe y la Federación Nacional de Cafeteros ha organizado un concurso conocido como "El premio Colombiano de Calidad de Café para Espresso" que es un reconocimiento para incentivar al caficultor colombiano en la producción de café de alta calidad. 
En el concurso pueden participar propietarios u organizaciones de propietarios, con un lote entre 15.000 y 30.000 kilos de café pergamino seco, que cumpla con las siguientes condiciones:

$\begin{array}{lll}\text { Humedad } & : & 10-12 \% \\ \% \text { Broca } & : & 1 \% \\ \% \text { Pasilla } & : & 5.5 \% \\ \text { FR } & : & 93 \\ \text { Taza } & : & \text { Limpia }\end{array}$

Los lotes de café son entregados a Alma café, en donde se obtendrá una muestra que se envía a la Oficina de Calidades en Bogotá para ser evaluada por esta oficina y posteriormente enviarla a Illycaffe, que es quien define los finalistas del concurso. La Cooperativa de Caficultores del Occidente de Nariño Ltda. Participó en el primer concurso de café para Espresso con las siguientes organizaciones de caficultores que se crearon a través de la Cooperativa:

Tabla 2. Organizaciones de caficultores de Nariño

\begin{tabular}{|c|c|c|c|c|}
\hline No. & Municipio & $\begin{array}{c}\text { Grupo / } \\
\text { asociación }\end{array}$ & Kilos & Representante \\
\hline 1 & Santa María & $\begin{array}{l}\text { Juanambú } \\
\text { Supremo }\end{array}$ & 30.000 & León Cabrera \\
\hline 2 & Sandoná & Café Dulce & 30.000 & Juvenal Melo \\
\hline 3 & Buesaco & La Curia & 17.000 & $\begin{array}{l}\text { Emelciro } \\
\text { Estrella }\end{array}$ \\
\hline 4 & Matituy-Tunja & Café florido & 20.400 & Gabriel Cañar \\
\hline 5 & Chachaguí & Sánchez & 21.000 & Javier Enríquez \\
\hline 6 & El Tambo & Tambo-Café & 24.640 & Libardo Criollo \\
\hline
\end{tabular}




\begin{tabular}{lll}
\hline TOTAL & 143.040 & Kilos
\end{tabular}

Fuente: Cooperativa de Caficultores del Occidente de Nariño Ltda.

Donde quedaron como finalistas los municipios de Sandoná, Chachagüí y El Tambo en los puestos Sexto, Séptimo y Octavo respectivamente, creando una nueva alternativa para el café del Occidente de Nariño y resaltando una vez más que se cuenta con la materia prima para participar en estos programas. Para el año 2.003 se realizó un trabajo más organizado en cuanto a la importancia de participar en "El segundo premio Colombiano de Calidad de Café para Espresso", apoyándonos en el Laboratorio de Calidades, y obteniendo mejores resultados. Se seleccionaron los lotes de acuerdo a sus características físicas y organolépticas más adecuados y se entró a participar con los siguientes grupos:

Tabla 3. Grupos del concurso cafetero

\begin{tabular}{cclcl}
\hline No. & Municipio & Grupo / asociación & Kilos & Representante \\
1 & Chachaguí & Sánchez & 15.000 & Amparo Maya \\
2 & Sandoná & Dulce Café & 15.143 & Esner Ibarra \\
3 & La Florida & La Florida & 14.465 & Focion \\
& & & & Martínez \\
4 & Matituy-Tunja & Café florido & 16.108 & Gabriel Cañar \\
\hline
\end{tabular}

Fuente: Cooperativa de Caficultores de Occidente de Nariño Ltda.

Gracias al trabajo integrado del Caficultor-Cooperativa-Laboratorio de Calidades, se logró obtener el primero tercero y décimo puesto en "El segundo premio Colombiano de Calidad de Café para Espresso" con los municipios de La Florida, Tunja-Matituy, y Sandoná, lo cual resalta el interés de la Cooperativa por participar de 
una manera más organizada en el programa de cafés especiales de la Federación Nacional de Cafeteros.

En el año 2.004 se organiza en Colombia El concurso de la Taza de excelencia, organizado por The Alliance for Coffe Excellence, Inc, buscando encontrar los mejores cafés de cada país. Se participó con 60 muestras de café de las cuales 15 fueron preseleccionadas por catadores expertos obteniendo 6 muestras finalistas, de las cuales se hizo acreedor al primer lugar premio el Señor Gregorio Arnulfo Botina del municipio de Sandoná, con un lote de 2000 kilos y en segundo lugar el Señor José Yepez del municipio de Chachaguí, confirmando una vez más la calidad del café en el Occidente del Departamento de Nariño. En el año 2008, se participó con 50 lotes de café de los cuales 27 pasaron a preselección, en esta ocasión el primer fue para la Finca Villa Loyola del Municipio de Chachaguí y de propiedad del la Compañía de Jesús obteniendo un puntaje de 92.39 .

Por otra parte, el ingeniero Oliva señala que considera a la Cooperativa un caso exitoso, por el cumplimiento que se ha tenido con el caficultor en lo relacionado con la comercialización del café pergamino seco, pagándole a un precio oportuno, con un precio justo, y un análisis de calidad justo; además, de brindarles otro tipo de servicios a los asociados. Visto de esta manera, son claras las garantías que los asociados tienen. Al respecto, el Ingeniero Oliva manifiesta:” tal es el éxito, que la cooperativa actualmente (año 2010) cuenta con dos mil asociados distribuidos en todos los municipios del occidente del Departamento de Nariño.

Es evidente, que detrás de un caso exitoso en una cadena productiva, existen una serie de actores que hacen posible el logro de unas metas. Al preguntarle al ingeniero Oliva sobre estos actores, el profesional enfatiza en los asociados, y señala que la 
cooperativa lo que hace es agruparlos y representarlos frente al Gremio cafetero en este caso la Federación Nacional de Cafeteros, que en Nariño está representada por el Comité Departamental.

Parte importante de un caso exitoso de una cadena productiva también son los estándares de calidad que se manejen; de tal manera, que el producto adquiera ciertas condiciones de competitividad que lo distingan de sus similares. Al preguntarle al Ingeniero Oliva: cómo ve la cadena productiva del café en Nariño con respecto a otras zonas cafeteras del país, el profesional dice:" Nariño más que todo se ha caracterizado y ha sobresalido su café, por su excelente calidad, hay unas condiciones climáticas y unas labores culturales que han hecho que el café de Nariño sea considerado como un café especial.

En Colombia, hay cuatro departamentos que están especializados en la comercialización de cafés especiales; de hecho, todo el café colombiano es de excelente calidad, pero hay cafés que sobresalen como es el caso de los cafés de Nariño, Cauca, Huila y Tolima, que por sus condiciones de calidad reciben sobreprecios; situación que estimula su producción y que al final se traduce en mejores ingresos para el caficultor.

Nariño es un gran productor de cafés especiales; señala el Ingeniero Oliva, que un $98 \%$ de los cafés producidos en Nariño, son considerados especiales; situación que se deriva de la gran labor de asesoría que cumple la Cooperativa de Caficultores del Occidente del Departamento de la mano con la Federación Nacional de cafeteros de Colombia y que como parte del éxito destaca la comercialización que hace la cooperativa con suiza y los Estados Unidos a quienes en este momento(año 2010) se les exporta aproximadamente 8 millones de kilos de café de alta calidad.

Otra parte importante de un caso exitoso de una cadena productiva, son las estrategias que se manejen para el logro de los objetivos. Al preguntarle al ingeniero 
Oliva, qué tipo de estrategias maneja la Cooperativa para lograr ese éxito, el profesional resalta, que la mejor estrategia son los servicios que la Cooperativa presta a sus asociados dadas las condiciones de competencia que se deriva del gremio; destaca, que parte de los servicios son la garantía de la compra, apoyada en un centro cercano al municipio que lo produce evitando así el desplazamiento del caficultor hacia la capital del Departamento.

Otro servicio que se presta es la garantía de un pesaje exacto que se apoya en básculas electrónicas certificadas bajo la supervisión del mismo caficultor, que encuentra en la atención personalizada de los funcionarios, amabilidad, servicio y confianza.

El análisis justo de calidad que la cooperativa presta a los asociados en su laboratorio, es otra estrategia para el logro del éxito. El manejo profesional que se hace a este proceso es de una gran responsabilidad señala el Ingeniero Oliva; de este análisis depende el precio que se va a cancelar y por ende el caficultor recibe lo que su café verdaderamente se merece con verdadera certeza.

Los auxilios educativos para los hijos de los asociados que se prestan en convenio con el ICETEX y que subsidian parte de las matrículas son parte de las estrategias del éxito alcanzado. Como también, ha sido la venta de fertilizantes de excelente calidad a un bajo precio y al cual se le suma la asistencia técnica de cómo utilizarlos y qué ajustes es preciso hacer en la finca productora.

Continuado con las estrategias utilizadas para el logro del éxito, el profesional Expresa:" Otra parte, es que les damos unos créditos; de la mano con la Federación de Cafeteros, en este momento hay un crédito que se llama Fertifuturo estos créditos han beneficiado mucho al productor por que ellos venden una parte de su café y en nueve 
meses lo entregan con cero intereses, si ellos ya compararon el abono después de marzo reciben un reintegro del $7.5 \%$ del valor que compraron su abono.

Dentro de esta dinámica en la que se mueve la Cooperativa de Caficultores del Occidente de Nariño Ltda., hablar de casos exitosos de esta cadena productiva, implica hacer un balance de resultados positivos y negativos. Al respecto, el Ingeniero Oliva señala que como resultados positivos son muchos y destaca la regulación del mercado que lo sustenta en los puntos de compra que tiene la Cooperativa en los diferentes municipios de occidente y que hace que los comerciantes no abusen del caficultor ofreciendo precios a su antojo, y que de la misma manera regula también los precios de los abonos.

Como aspectos negativos el profesional expresa que en la cooperativa existen muchas debilidades como en toda empresa. Destaca que la Cooperativa se está quedando en el plano de ser una empresa únicamente comercializadora de materia prima; de allí que la propuesta para próximos años dentro de sus puntos de planeación, es entrar al procesamiento del café con una marca propia que permita la exportación directa de cafés especiales situación que desde ya evidencia grandes augurios para la Cooperativa.

En este sentido y apuntando al proyecto de entrar a competir en el mercado del café con una marca propia, es preciso resaltar la importancia del uso de nuevas tecnologías por parte de los caficultores como actores de la cadena productiva y en razón de que la Cooperativa es uno de los principales centros de acopio del producto y será la organización la encargada de garantizar un producto de alta calidad sobre la base de buenas prácticas agrícolas y de manufactura.

Al respecto, al preguntarle al ingeniero Oliva sobre la pertinencia y la importancia de estos procesos, dice:" De hecho las buenas prácticas agrícolas ya se 
están aplicando y es lo más importante por que en toda la cadena se debe realizar unas buenas prácticas agrícolas y de manufactura en aras de un proceso de certificación que ya está en marcha y por otro lado, por que los clientes que tenemos nos exigen estas prácticas que se aplican al cultivo, el proceso de beneficio, el manejo del agua e inclusive el pago justo a los trabajadores. Es de aclarar que esto lo está haciendo la Federación de cafeteros a través de unos técnicos del programa en Expresso. La Cooperativa por su parte, está en un proceso de visita de fincas, certificación a los asociados paralelo a lo que hace la federación y que se realiza con un grupo de trabajo propio de la Cooperativa.

Parte de los fundamentos de este caso exitoso, es que los compradores de café son fieles de compras, ellos son empleados directos que trabajan todo el año; por lo tanto, en la época de cosecha entre mayo y agosto se dedican a la compra de café y el resto del tiempo se dedican a trabajos de campo ofreciendo asesoría técnica.

Destaca el profesional, que una parte negativa y que entorpece el desarrollo de estas prácticas, es la parte económica de algunos caficultores que se evidencia en la carencia de herramientas como despulpadoras y patios de secado, sustentando así la importancia de dotar al caficultor nariñense de nuevas tecnologías que le permitan mejorar aún más la calidad del producto.

Dato importante para resaltar es que de acuerdo a la Cooperativa de caficultores del departamento de Nariño: hoy existen más de 20 mil familias productoras con 30 mil ha. Sembradas con café arábigo. Por su importancia social y económica esta actividad es considerada motor del desarrollo regional y uno de los pilares de la cultura y el tejido social Nariñense. Visto de esta manera, la producción del café en el Departamento, genera una cantidad considerable de fuentes de trabajo. Parte relevante de este caso exitoso es el compromiso que la Cooperativa de caficultores del Occidente del Nariño 
tiene con las familias caficultoras al no permitir el uso de mano de obra en menores de dieciocho años.

Algunos productores sostienen que deberá corregirse algunas debilidades como la pobreza de los mismos cafeteros que choca con la sostenibilidad, la ineficiencia de la utilización del agua por no existir controles en la utilización del recurso y el tratamiento después de la utilización del mismo; ¿entonces, la pregunta que surge es cómo aumentar la sostenibilidad del café?

Al respecto el Ingeniero Oliva expresa, que, si bien es cierto que la Federación Nacional de cafeteros ya está trabajando sobre el tema, la verdad es que se ha encontrado alguna resistencia a los cambios que esto implica, sinembargo la utilización de trampas de grasa en los beneficiaderos que impiden gran parte de la contaminación del agua, ya se visualizan en una gran mayoría de las fincas cafeteras contribuyendo de manera significativa a la conservación del medio ambiente.

Para la Cooperativa el café llegó a Nariño en el siglo XIX, su cultivo se fue esparciendo por los ricos suelos volcánicos que cubren la región, la caficultura Nariñense encontró la riqueza ambiental que permitió distinguirse por el gran tamaño de su grano, su suave sabor y su excelente calidad.

Destaca también que en Nariño existen 2 grandes zonas cafeteras; la del norte y la de occidente, considerados como una caficultura de alta adopción, de buenas prácticas y renovación; su promedio de edad es de 5 años, lo que la convierte en una de las más competitivas del País. Desde este panorama, el caficultor nariñense debería obtener una buena rentabilidad. Sin embargo, la Federación Nacional de Cafeteros de Colombia en un artículo del periódico la República en la edición del 27 de junio de 2007, sostiene que menos del $10 \%$ de la millonaria renta que genera la industria mundial del café, termina en manos de los productores; de allí la preocupación de la 
cooperativa por incentivar a los caficultores nariñenses a concentrarse más en la calidad que en la cantidad por que como se señaló anteriormente la calidad se paga en Nariño.

Otro aspecto importante de destacar en este caso exitoso de la cadena productiva del café, es la preocupación que la Cooperativa tiene por cambiar la mentalidad del agricultor de campesino a empresario. Carlos Oliva sostiene que es un tema que se está trabajando por que es preciso que el caficultor tenga sus cuentas claras con respecto a los gastos de producción y a las ganancias que obtiene y para eso es preciso capacitarlos.

En síntesis, este breve análisis sobre un caso exitoso de la cadena productiva del café en el departamento de Nariño y cuyo protagonista es la Cooperativa de Caficultores del Occidente de Nariño Ltda., nos invita a reflexionar sobre la importancia del desarrollo estratégico que la organización ha tenido, y que indudablemente se enfocan hacia un acertado servicio al cliente y al permanente esfuerzo por propender por el mejoramiento de la calidad del café en Nariño.

De continuar con esta clara visión, los casos exitosos para esta Cooperativa serán más y se verán venir a muy corto plazo promoviendo la prosperidad económica y social de los asociados y sus familias y el desarrollo económico regional como lo da a entender el objetivo de su plataforma estratégica. Buen tiempo y buena mar para la Cooperativa.

\section{Conclusiones}

Punto a destacar en este caso exitoso de la cadena productiva del café en Nariño, son las estrategias que se manejen para el logro de los objetivos y que particularmente se concentran en los servicios que la Cooperativa presta a sus asociados dadas las condiciones de competencia que se deriva del gremio; destaca, que parte de los servicios 
son la garantía de la compra, apoyada en un centro cercano al municipio que lo produce evitando así el desplazamiento del caficultor hacia la capital del Departamento.

Por otro lado, es preciso destacar la preocupación que la Cooperativa tiene por cambiar la mentalidad del agricultor de campesino a empresario. Se sabe que es un tema que se está trabajando porque es preciso que el caficultor tenga sus cuentas claras con respecto a los gastos de producción y a las ganancias que obtiene y para eso es preciso capacitarlos.

Este breve análisis nos invita a reflexionar sobre la importancia del desarrollo estratégico que la organización ha tenido, y que indudablemente se enfocan hacia un acertado servicio al cliente y al permanente esfuerzo por propender por el mejoramiento de la calidad del café en Nariño.

\section{Bibliografía}

Café Kina \& Ko (sf.). Historia del cafeto. Recuperado de: http://cafekina.com/Sp/HistoriaDelCafe.htm

Comisión de Ajuste de la Institucionalidad Cafetera. (2002). Resumen ejecutivo.

Recuperado de:

http://www.destinocafe.com/es/ieventos/ver/59/la_excepcionalidad_de_nuestro_ paisaje_cafetero/

Cooperativa de Caficultores del Occidente de Nariño Ltda. Recuperado de: http://www.informacion-empresas.co/Empresa_COOPERATIVA-

CAFICULTORES-OCCIDENTE-NARINO-LTDA.html.

Federación Nacional de Cafeteros. (2010) Una bonita historia. Recuperado de: http://www.cafedecolombia.com/particulares/es/el_cafe_de_colombia/una_bonit a_historia/ 
La hora nacional. (2008). Internacionalizarán el café de Nariño. Recuperado de: http://www.lahora.com.ec/index.php/noticias/show/671872/-

1/Internacionalizar\%C3\%A1n_el_caf\%C3\%A9_de_Nari\%C3\%B10.html\#.UcD $\underline{\mathrm{hX} 9 \text { ihjxU }}$

Oliva, C. (2004) Fiel de Compras Sede Pasto, analista de Calidad, y catador de café certificado como Juez de Catación por la Asociación de Cafés Especiales de América (SCAA) en Long Beach California, en el año 2004. 